\title{
Modélisation physique de fondations et d'ouvrages maritimes
}

\author{
GÉrard Raulta ${ }^{\mathrm{a}}$, Luc Thorel et Jacques Garnier \\ Laboratoire Central des Ponts et Chaussées, Division Reconnaissance et Mécanique des Sols, Section Mécanique des Sols et \\ Centrifugeuse, Route de Bouaye, BP 4129, 44341 Bouguenais Cedex, France
}

Reçu le 17 décembre 2004, accepté le 13 octobre 2005

\begin{abstract}
Résumé - Après avoir rappelé les principes de la modélisation physique en centrifugeuse, technique aujourd'hui très répandue, plusieurs exemples d'expérimentations réalisées au LCPC, sur des modèles réduits de fondations et d'ouvrages maritimes sont présentés. Ces études sont le plus souvent entreprises pour contribuer au dimensionnement d'ouvrages réels (plates-formes offshore, ponts, quais...). Elles nécessitent la reconstitution de massifs de sol de mêmes caractéristiques mécaniques que celles des fonds marins. Ces expérimentations sont validées par deux méthodes différentes : modélisation à différentes échelles ou comparaison avec des observations faites sur ouvrages en vraie grandeur. Les cas présentés portent, entre autres, sur les fondations profondes, les ancrages à effet de succion, les ouvrages de soutènement et les fondations sur sols renforcés.
\end{abstract}

Mots clés : Modèle physique / centrifugeuse géotechnique / fondation / validation

Abstract - Physical modelling of off-shore foundations and structures. After a brief summary of centrifuge physical modelling, technique widely used today, several examples of research programmes performed in LCPC on reduced scale models of foundations and offshore structures are presented. These studies are often performed to contribute to the design of prototype structures (offshore platforms, bridges, piers,...). The reconstitution of soil samples with the same mechanical characteristics of marine soils is then required. The centrifuge modelling technique may be validated with two different approaches: modelling at different reduced scales or observations done on prototype structures. This paper describes experiments on deep foundations, suction anchors, retaining structures and foundations embedded in reinforced soils.

Key words: Physical modelling / geotechnical centrifuge / foundation / validation

\section{Introduction}

Parmi les méthodes de modélisation utilisées en géotechnique en vue du dimensionnement des ouvrages, les essais sur modèles réduits testés en macrogravité viennent en complément de la modélisation numérique (éléments finis, analyse limite, ... ) et des essais sur site réels. Nous traçons un tour d'horizon des expérimentations réalisées au LCPC sur modèles réduits centrifugés dans le domaine du génie maritime et côtier.

\section{Modélisation physique en centrifugeuse}

Les modèles réduits permettent, en suivant certaines règles de similitude, de reproduire à une échelle réduite certains aspects du comportement des ouvrages en vraie

a Auteur correspondant : Gerard.Rault@lcpc.fr grandeur. En géotechnique, ces règles imposent, si le modèle est à l'échelle $1 / n$, qu'il soit testé sous une accélération $n$ fois supérieure à la pesanteur terrestre. Plusieurs solutions ont été imaginées pour satisfaire cette condition de similitude mais la centrifugation du modèle s'est largement imposée depuis une quinzaine d'années en raison de l'étendue de ses domaines d'application $[1,2]$.

On recense actuellement 15 installations en service en Europe, autant aux USA, une trentaine en Asie et une en Afrique (Université Mansoura en Égypte). On dénombre par ailleurs plus d'une trentaine de centrifugeuses de rayon supérieur à $3 \mathrm{~m}$ (Tab. 1).

Dès le début des années 1970, le LCPC s'est intéressé à cette méthode [3] et dispose d'une installation depuis fin 1985 [4]. D'un rayon de $5,5 \mathrm{~m}$, elle peut soumettre un modèle de 2 tonnes à une accélération 100 fois supérieure à la pesanteur terrestre (Fig. 1). Entourée d'équipements spécifiques aux essais en macrogravité [5], elle a récemment été équipée d'un robot (Fig. 1) qui 
Tableau 1. Centrifugeuses géotechniques de rayon $R$ supérieur ou égal à $3 \mathrm{~m}$. En gras sont repérées les centrifugeuses réalisées sur le modèle de celle du LCPC par la société Actidyn Systèmes (anciennement Acutronic). $G_{\max }=$ accélération maximale, $M_{\max }=$ masse embarquée maximale.

\begin{tabular}{|c|c|c|c|c|c|}
\hline Centre/Ville & Pays & Année & $\begin{array}{l}R \\
{[\mathrm{~m}]}\end{array}$ & $\begin{array}{l}G_{\max } \\
{[\mathrm{g}]}\end{array}$ & $\begin{array}{l}M_{\max } \\
{[\mathrm{t}]}\end{array}$ \\
\hline CEA-CESTA/Le Barp & FR & 1964 & 10,5 & 100 & 2 \\
\hline NIISISM/Baku & $\mathrm{SU}$ & 1966 & 5,5 & 100 & \\
\hline Sandia University/Albuquerque & US & 1968 & 7,6 & 200 & 3,5 \\
\hline Simon Eng. Lab./Manchester & UK & 1971 & 3,2 & 140 & 5,5 \\
\hline CUED/Cambridge & UK & 1972 & 4,3 & 150 & 1 \\
\hline AZNIISM/Baku & SU & 1974 & 5,5 & 500 & 2,5 \\
\hline Port \& Harbour Research Institute/Yokosuka & JP & 1980 & 3,8 & 113 & 2,76 \\
\hline Yangtze River Scientific Research Institute/Wuhan & $\mathrm{CN}$ & 1983 & 3 & 300 & 0,5 \\
\hline Chengdu Hydroelectric Investigation \& Design Institute/Chengdu & $\mathrm{CN}$ & 1985 & 5,4 & 100 & 3 \\
\hline LCPC/Nantes & FR & 1985 & 5,5 & 200 & 2 \\
\hline Rhur Unviversität/Bochum & $\mathrm{DE}$ & 1987 & 4,1 & 250 & 2 \\
\hline Univ. California/Davis (California) & US & 1987 & 9,14 & 300 & 3,64 \\
\hline Univ. Colorado/Boulder & US & 1987 & 5,49 & 200 & 2 \\
\hline Chuo University & $\mathrm{JP}$ & 1988 & 3,05 & 150 & 0,66 \\
\hline GeoDelft & NL & 1988 & 6 & 500 & 5,5 \\
\hline Rensselaer Polytechnic Institute/Troy (New York) & US & 1989 & 3 & 200 & 1 \\
\hline Kajima Co./Chofu-Tokyo & $\mathrm{JP}$ & 1990 & 3 & 200 & 1 \\
\hline $\begin{array}{l}\text { Institute Water Conservancy \& Hydroelectric } \\
\text { Power Research/Beijing }\end{array}$ & $\mathrm{CN}$ & 1991 & 5,03 & 300 & 1,5 \\
\hline $\begin{array}{l}\text { Nanjing Hydraulic Research Institute Geotechnical } \\
\text { Centrifuge Center }\end{array}$ & $\mathrm{CN}$ & 1991 & 5,5 & 200 & 2 \\
\hline Shimizu Co. & JP & 1991 & 3,35 & 100 & 0,75 \\
\hline Nakase Nikken Sekkei Institute/Kawasaki & $\mathrm{JP}$ & 1992 & 3 & 200 & 1 \\
\hline Center for Cold Ocean Resources Engineering/St John's & CA & 1993 & 5,5 & 200 & 2,2 \\
\hline Takenaka Komuten/Chiba-Tokyo & JP & 1993 & 6,5 & 200 & 5 \\
\hline $\begin{array}{l}\text { US Army Corps of Engineers-Waterways } \\
\text { Experiment Station/Vicksburgh (Miss.) }\end{array}$ & US & 1993 & 6,5 & 350 & 6 \\
\hline Fisheries Agency & JP & 1994 & 3 & 150 & 0,25 \\
\hline National Central Univ/Chung-li & TW & 1995 & 3 & 200 & 0,55 \\
\hline Nishimatsu Construction Co./Kanagawa & JP & 1997 & 3,8 & 150 & 1,28 \\
\hline Public Works Research Institute, Ministry of Construction/Tsukuba & JP & 1997 & 6,6 & 150 & 5 \\
\hline Daewoo Institue of Construction Technology/Suwon & $\mathrm{KR}$ & 1997 & 3 & 100 & 1,2 \\
\hline Dundee University/Dundee & UK & 1999 & 3,5 & 130 & 0,8 \\
\hline Hong Kong University of Science and Technology/Kowloon & HK & 2000 & 4,47 & 150 & 4 \\
\hline Technical Research Institute of Obayashi/Tokyo & JP & 2000 & 3,5 & 120 & 7 \\
\hline Mansoura University/Alexandria & EG & 2001 & 3,5 & 130 & 0,8 \\
\hline IIESS Téhéran (projet) & IR & 2004 & 3,5 & 130 & 0,8 \\
\hline Téhéran Univ. (projet) & IR & 2004 & 3,5 & 130 & 0,8 \\
\hline
\end{tabular}

permet d'enchaîner différentes opérations sur le modèle sans arrêt de la machine. Son taux d'occupation est supérieur à 200 jours par an, une série d'expérimentations différentes étant programmée chaque semaine.

Pratiquement tous les types d'ouvrages géotechniques sont susceptibles d'être étudiés sur modèles réduits comme on peut le constater en consultant les actes des congrès internationaux « Centrifuge » périodiquement organisés en 1988 [6], 1991 [7], 1994 [8], 1998 [9] et 2002 [10]. L'examen des programmes d'études et de recherches conduits au LCPC depuis 1986 est une autre source d'information sur les domaines actuellement couverts (Fig. 2). Les études de fondations superficielles et profondes représentent à elles seules plus du tiers du temps d'utilisation de la centrifugeuse, suivies des renforcements de sols, des soutènements, des tunnels, des ouvrages offshore, des sols compressibles.

On assiste cependant à une évolution de l'activité, conséquence des progrès techniques qui autorisent des opérations de plus en plus complexes sur le modèle en cours de rotation. La centrifugeuse du LCPC sera par exemple prochainement dotée d'un générateur de tremblement de terre permettant d'étudier la réponse des ouvrages aux sollicitations sismiques.

Comme toute nouvelle approche, la modélisation physique en centrifugeuse demande à être validée. Une première méthode (modelling of models) consiste à reconduire les expérimentations simulant le même prototype à des échelles de réduction différentes. Si la similitude est respectée, les résultats obtenus à ces différentes échelles 

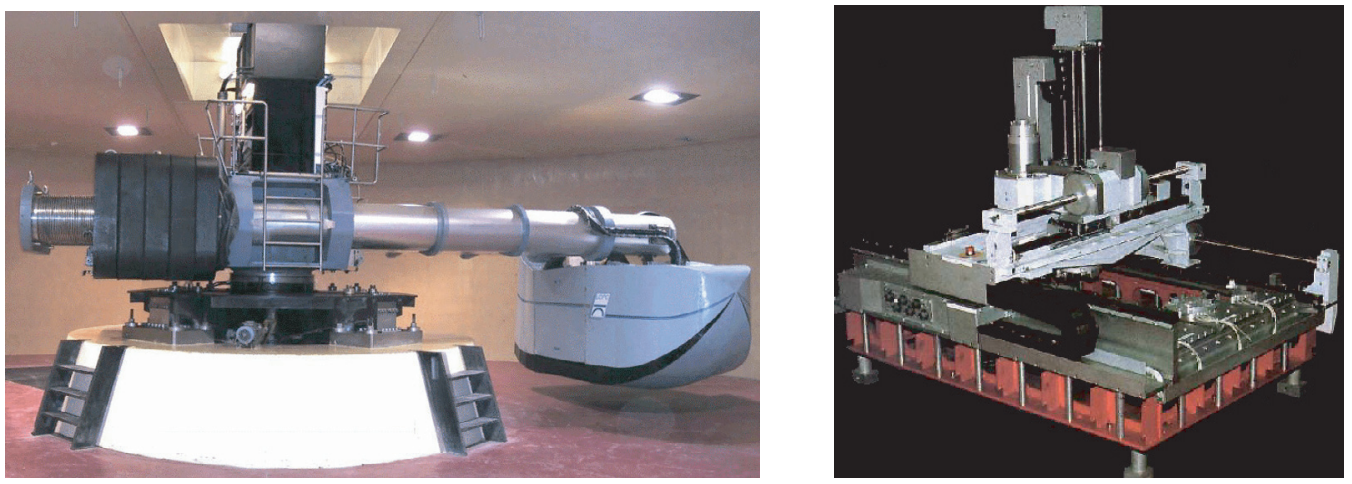

Fig. 1. Centrifugeuse géotechnique (rayon $5,5 \mathrm{~m}$ ) et téléopérateur du LCPC.

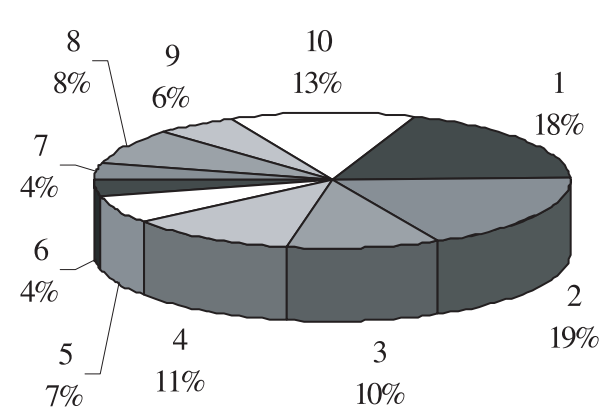

\begin{tabular}{|c|c|} 
Symbole & Ouvrage étudié \\
\hline 1 & Fondations superficielles \\
2 & Fondations profondes \\
3 & Ancrages d'ouvrages offshore \\
4 & Renforcements et soutènements \\
5 & Tunnels en terrain meuble \\
6 & Sols compressibles \\
7 & Matériaux granulaires \\
8 & Divers problèmes géotechniques \\
9 & Essais hors génie civil \\
10 & Etude des conditions de similitude
\end{tabular}

Fig. 2. Répartition par type d'ouvrage géotechnique des études sur modèles réduits conduites au LCPC depuis 1986 [1].

doivent être identiques. Dans la seconde méthode, on compare les résultats d'essais sur modèles aux données obtenues sur des ouvrages en vraie grandeur. On trouvera dans [1] les résultats de ces comparaisons pour des fondations superficielles et profondes, des caissons à effet de succion, des essais au pénétromètre, de la consolidation des vases.

\section{Pieux sous charge latérale}

Les méthodes en déplacement (courbes de réaction $p-y)$ se sont généralisées dans la majorité des règlements. Dans la pratique et suivant les pays, les courbes $p-y$ sont déduites des résultats d'essais de laboratoire ou d'essais en place. Des recherches se sont avérées nécessaires pour combler les lacunes importantes qui subsistent dans les règles actuelles (effets de groupe, proximité d'un talus, chargement cyclique).

Une méthode expérimentale a été d'abord développée permettant de déduire les courbes de réaction $p-y$ des profils de moments de flexion dans les pieux. Une vingtaine de paires de jauges équipe les pieux permettant la mesure des moments. Ces données $M(z)$ sont ensuite lissées par des fonctions splines quintiques (logiciel SLIVALICS-LCPC). Les déplacements $y(z)$ et les pressions $p(z)$ sont obtenus respectivement par double intégration et double dérivation des fonctions $M(z)$. Les courbes $p-y$ sont enfin validées en les réintroduisant dans le logiciel de calcul des pieux sous charge latérale (PILATE-LCPC) et la réponse du pieu calculée est comparée aux données expérimentales (Fig. 3).
Après validation, les courbes $p-y$ déterminées expérimentalement peuvent être analysées et les programmes suivants ont été engagés dans le cadre de différentes thèses :

- Étude des charges limites et des courbes $p-y$ sous grands déplacements [11]

- Effet de la proximité d'un talus sur les courbes de réactions $p-y[12]$

- Effet de groupe sur les courbes de réaction $p-y[13]$

- Réponse d'un pieu sous un choc latéral en tête [14]

- Effet de charges cycliques sur les courbes $p-y$ [15].

\section{Ancrages à effet de succion}

Dix pour cent de l'activité de la centrifugeuse du LCPC sont consacrés aux ouvrages off-shore, toujours dans le cadre de contrats. Les bureaux d'étude et les compagnies pétrolières ont rapidement vu l'intérêt des modèles réduits et les besoins de recherche se sont en outre accru avec le début de l'exploitation de champs en mers profondes (1000 m à plus de $2000 \mathrm{~m}$ d'eau). Des solutions innovantes doivent donc être recherchées pour l'ancrage des plates-formes (caissons à succion, pieux avec point d'ancrage à grande profondeur, pieu à inertie variable, plaques pivotantes). Le tableau 2 présente les principales études sur modèles conduites au LCPC dans le domaine des fondations des ouvrages maritimes et off-shore. Comme le montre ce tableau, le nombre de publications relatant ces travaux est extrêmement réduit eu égard à 

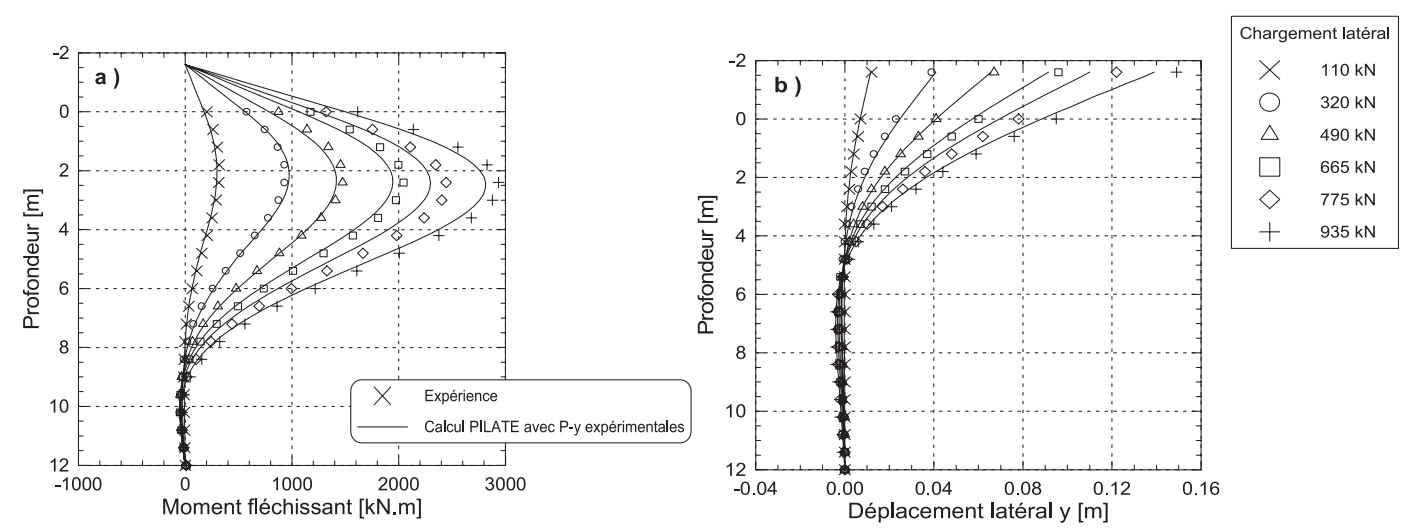

Fig. 3. Validation des courbes $p-y$ expérimentales [13].

Tableau 2. Principaux programmes sur les fondations d'ouvrages maritimes et off-shore réalisées au LCPC depuis 1990.

\begin{tabular}{|l|l|l|l|}
\hline Contrats & Dates & Objet & Référence \\
\hline Exxon EPR & 1989 à 1992 & $\begin{array}{l}\text { Caissons } h=30 \mathrm{~m}, \emptyset=15 \mathrm{~m} \text { sous charges } \\
\text { verticales statiques et cycliques }\end{array}$ & {$[17-19]$} \\
\hline Exxon EPR & 1993 & $\begin{array}{l}\text { Modélisation d'essais sur caisson à } \\
\text { succion réalisés sur site par le NGI }\end{array}$ & {$[20,21]$} \\
\hline BOS - Geodia & $1991-1992$ & Plancher de stabilité à effet de succion & Rapport \\
\hline BOS - Geodia & $1994-1995$ & Groupe de 9 pieux et fondations mixtes & Rapport \\
\hline $\begin{array}{l}\text { Ifremer } \\
\text { Geodia }\end{array}$ & 1995 & $\begin{array}{l}\text { Fondations à effet de succion sous } \\
\text { charges statiques et cycliques }\end{array}$ & {$[22]$} \\
\hline $\begin{array}{l}\text { Ifremer } \\
\text { Geodia }\end{array}$ & 1995 & $\begin{array}{l}\text { Groupes de } 3 \text { pieux pour tour souple - } \\
\text { Détermination des raideurs horizontale et } \\
\text { verticale du système de fondation }\end{array}$ & Rapport \\
\hline Chevron & 1996 & $\begin{array}{l}\text { Pieux d'ancrage de la plate-forme Genesis } \\
\text { (L=76 m, B = 2,44 m) }\end{array}$ & Rapport \\
\hline GDS - Vinci & 1999 & $\begin{array}{l}\text { Fondations du pont Rion-Antirion } \\
\text { (charges limites, cycles d'effort latéral et } \\
\text { de moments de renversement) }\end{array}$ & {$[23,24]$} \\
\hline Ifremer- Sage-Geodia & 2000 à 2001 & Caissons à effet de succion & Rapport \\
\hline Exxon Mobil & 2000 à 2003 & $\begin{array}{l}\text { Caissons à effet de succion (effet du mode } \\
\text { de fonçage, résistance aux charges de } \\
\text { longue durée et aux cycles) }\end{array}$ & Rapport \\
\hline Université Grenoble & $2002-2003$ & Plaques à effet de succion & Rapport \\
\hline
\end{tabular}

l'ampleur des programmes qui ont parfois nécessité plusieurs années d'études. La raison en incombe aux clauses de confidentialité drastiques souvent imposées dans ce type de contrat.

Pour les caissons à effet de succion, les dispositifs développés (Fig. 4) permettent de reproduire à la fois la phase de mise en place (avec application d'une succion active sous la tête) puis la phase de chargement (sollicitations cycliques simulant l'effet de la houle, des courants ou des tempêtes) sans arrêt de la centrifugeuse. Les fortes succions passives qui sont générées confèrent à ces ancrages des capacités de résistance énormes. Les ruptures sous charge verticale (Tension Leg Platform) sont de type capacité portante inverse (reverse bearing capacity).

La figure 5 montre les caissons utilisés lors d'une importante étude réalisée pour le centre de recherche d'Exxon Mobil [16].
Un exemple d'étude d'ancrage par pieux concerne la plate-forme Genesis (Chevron) installée en 1999 dans le Golfe du Mexique (site Red Canyon). De type SPAR, cette plate-forme de $198 \mathrm{~m}$ de hauteur flotte dans près de $800 \mathrm{~m}$ d'eau et est ancrée sur 14 pieux (fiche de $67 \mathrm{~m}$, diamètre de 2,44 $\mathrm{m}$ ) répartis sur un cercle de $1600 \mathrm{~m}$ de diamètre (Fig. 6).

Les efforts inclinés à $37^{\circ}$ sur l'horizontale sont appliqués sur les pieux à une profondeur dans le sol de $17 \mathrm{~m}$. À la demande de Chevron, une étude sur modèles a été réalisée sur la réponse de ces pieux à des programmes de charges statiques et cycliques, verticales et inclinées allant jusqu'à rupture. Elle a permis de concevoir des pieux à inertie variable en augmentant les sections d'acier dans les zones de moments les plus forts. Une modélisation à différentes échelles du même prototype a également permis de vérifier les lois de similitude. 


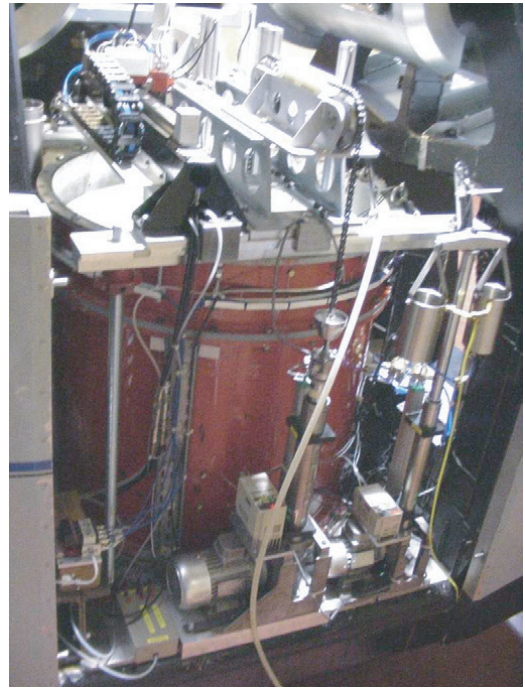

a-Vue latérale

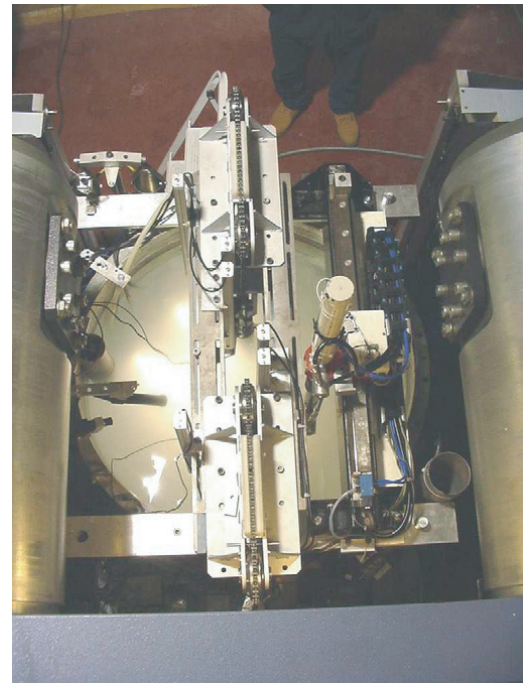

b-Vue de dessus

Fig. 4. Dispositif d'essai sur les caissons à effet de succion.

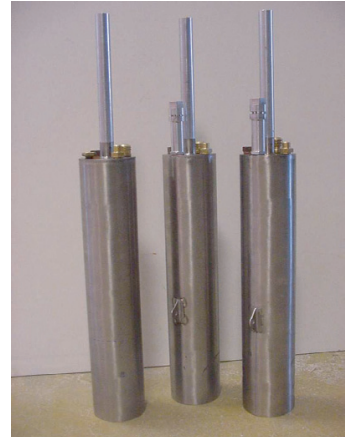

(a) Caissons modèles à

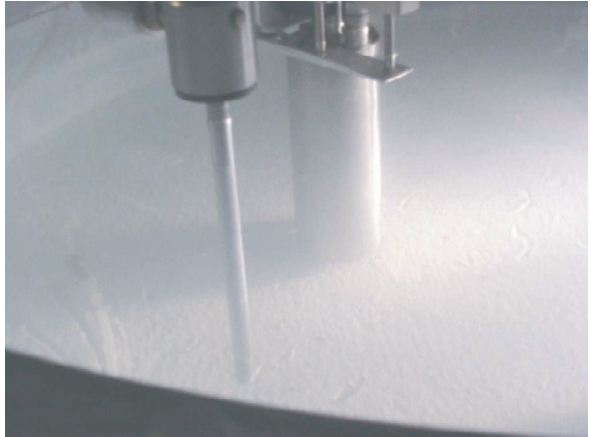

(b) Caisson immerge avant fonçage dans le massif de sol

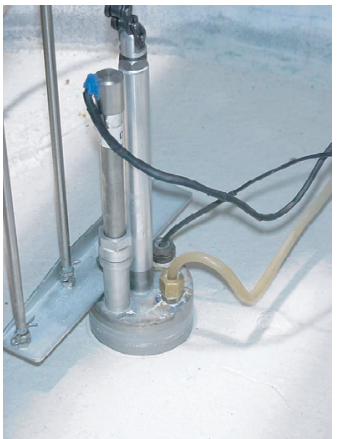

(c) Caisson en place avant l'essai de chargement

Fig. 5. Modèles réduits de caisson à effet de succion [16]

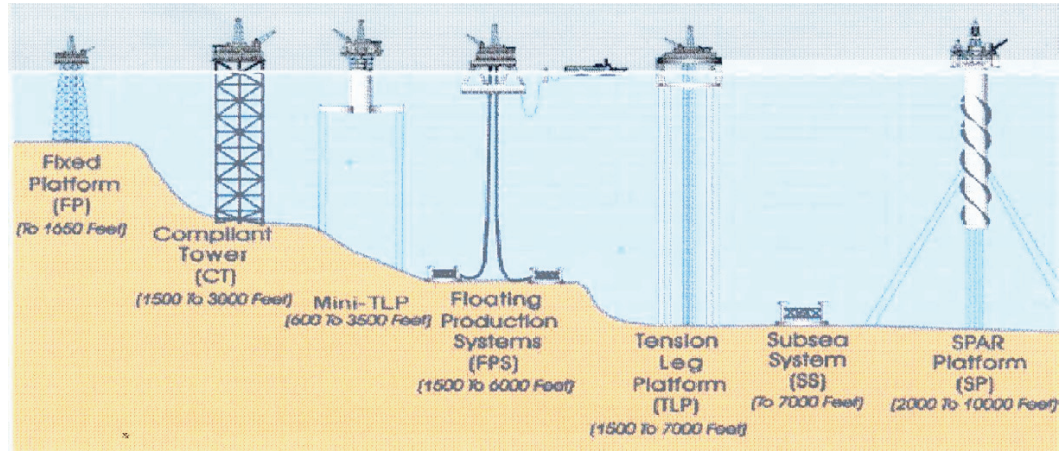

Fig. 6. Évolution des fondations des plates-formes off-shore avec la hauteur d'eau.

\section{Fondation du pont Rion-Antirion sur un sol renforcé}

Un ouvrage très innovant vient d'être mis en service en Grèce près de Patras, à 250 km à l'ouest d'Athènes [24,25]. Ce pont repose sur quatre piles et la travée centrale a une longueur de $560 \mathrm{~m}$. Le poids total d'une pile est de l'ordre de $800 \mathrm{MN}$ et elle doit résister à un effort horizontal de
$600 \mathrm{MN}$ et un moment de renversement de 20000 MN.m. La conception des fondations a été rendue complexe par les conditions du site (plus de $500 \mathrm{~m}$ de sols médiocres, très forte sismicité, risque de collision de tankers, hauteur d'eau de $65 \mathrm{~m}$, vent de $200 \mathrm{~km} \cdot \mathrm{h}^{-1}$ ). Le système de fondation des piles conçu par Géodynamique et Structure (GDS) comprend un renforcement du sol en place par 250 tubes métalliques verticaux de $2 \mathrm{~m}$ de diamètre 


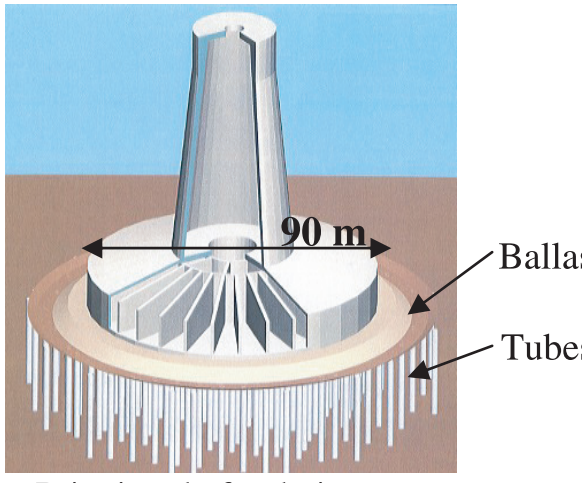

a-Principe de fondation

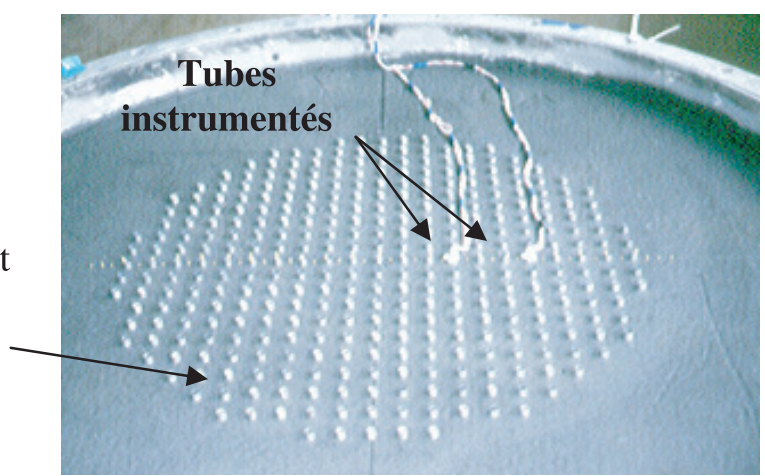

b-Modèle $\left(1 / 100^{\text {ème }}\right)$ du sol renforcé par inclusions avant mise en place de la couche de ballast

Fig. 7. Fondation des piles du pont Rion-Antirion.

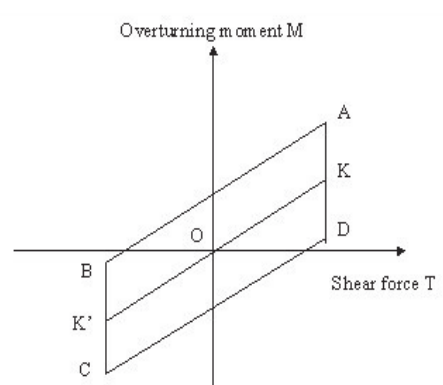

Fig. 8. Programmes de chargement cyclique appliqués aux modèles.

et $30 \mathrm{~m}$ de fiche, la mise en place d'une couche de ballast de $5 \mathrm{~m}$ d'épaisseur puis la construction de la fondation proprement dite, de $90 \mathrm{~m}$ de diamètre (Fig. 7).

Une campagne d'essai sur modèles a été entreprise pour valider ce système de fondation composite. L'argile du site a été utilisée et la figure 7 montre l'un des modèles après renforcement par les tubes métalliques et avant mise en place de la couche de ballast $[22,23]$. Certains tubes étaient instrumentés de jauges de déformation pour la détermination des moments de flexion. Les pressions interstitielles étaient mesurées à l'aide de sondes mises en place dans le massif d'argile.

Les programmes de chargement cyclique combinaient un effort latéral $T$ et un moment de renversement $M$ suivi d'un chargement monotone jusqu'à la rupture (Fig. 8). La série d'essais sur modèles et l'étude paramétrique ont permis de vérifier le bon comportement du système de fondation sous charge de service, de mesurer ses capacités portantes limites (Fig. 9), de déterminer les mécanismes de rupture (et de les comparer avec les mécanismes théoriques retenus lors de la conception et du calcul des fondations) et même d'optimiser le nombre d'inclusions verticales nécessaires au renforcement du sol de fondation.

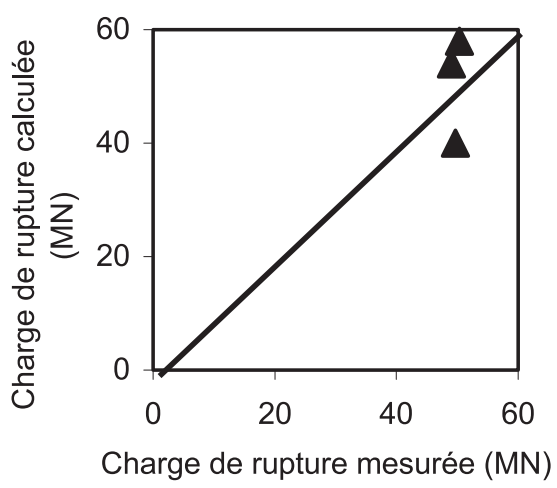

Fig. 9. Comparaison entre charges de rupture calculées et mesurées.

\section{Soutènements}

La validation des méthodes théoriques ou numériques repose essentiellement sur la comparaison des résultats des calculs avec des mesures sur sites. Cependant ces comparaisons sont souvent délicates à mener, en raison des conditions expérimentales (précision des mesures, variabilité des paramètres de sol, chargement...) mais aussi des limites des modèles numériques (lois de comportement, modélisation des interfaces sol-structure... ). L'un des avantages de la modélisation physique réside dans une meilleure maîtrise des procédures expérimentales, des caractéristiques des matériaux et des dimensions du modèle.

Une étude de ce type a été menée récemment sur un écran de soutènement autostable de $10 \mathrm{~m}$ de hauteur, fiché dans un sable sec, et de son interaction avec une fondation avoisinante [26]. Cette configuration est souvent rencontrée lors de la conception de quais. La modélisation de ce type d'ouvrage est possible depuis peu grâce à l'utilisation du téléopérateur qui permet de simuler (par ratissage) un processus d'excavation réel en cours de centrifugation [27]. Le dispositif expérimental permet le suivi, au cours de l'excavation, des moments de flexion dans la paroi, des déplacements horizontaux de la paroi, des tassements du sol soutenu, ainsi que des déplacements horizontaux et verticaux de la fondation. 


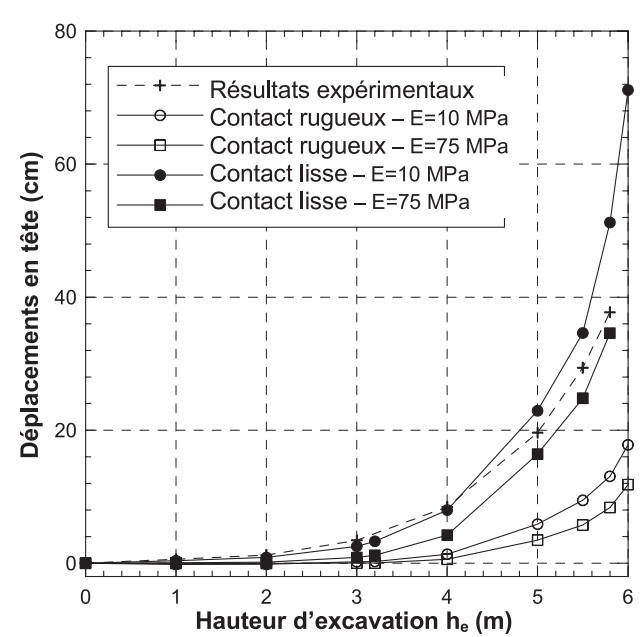

Fig. 10. Comparaison de résultats d'essais et de calculs par éléments finis. Effets de la nature de l'interface et du module élastique (modèle de Mohr-Coulomb).

Les données expérimentales recueillies sont comparées aux résultats de modélisations numériques utilisant le code CESAR-LCPC avec deux modèles de comportement, Mohr-Coulomb et Vermeer [28]. Le premier est couramment utilisé et ses paramètres ont été déterminés à partir d'essais triaxiaux conventionnels. Le second est un modèle isotrope, élastique non-linéaire, plastique, à deux surfaces de charge, particulièrement bien adapté aux sables denses. Ses paramètres sont déterminés à partir d'essais triaxiaux particuliers [29] conduits sur des chemins de contraintes spécifiques, représentant les chemins de contraintes existant aux abords d'un ouvrage de soutènement.

La comparaison des résultats expérimentaux et des résultats des calculs numériques pour différentes configurations (hauteur d'excavation, rigidité de la paroi, distance paroi fondation) a permis de déterminer la loi de comportement et les paramètres les mieux adaptés à ce type de problème ainsi que les spécificités de modélisation de ce type d'ouvrage (nature d'interface, initialisation du champ de contraintes...). Il apparaît ainsi que l'utilisation du modèle de Mohr-Coulomb nécessite l'emploi artificiel d'une interface sol-paroi totalement lisse afin de limiter les effets du soulèvement du fond de fouille engendré par la partie élastique du modèle lors du déconfinement (Fig. 10). L'emploi du modèle de Vermeer permet de s'affranchir de ce biais et de modéliser l'interface selon ses caractéristiques physiques réelles.

Les résultats montrent également qu'il est nécessaire d'adapter les paramètres de sol aux différentes phases de l'excavation. Lors des premières phases de l'excavation, le comportement de l'ouvrage est en effet principalement gouverné par la poussée exercée sur la partie hors fiche. Les essais triaxiaux menés sur ces chemins spécifiques font apparaître un module élastique de l'ordre de $10 \mathrm{MPa}$. À l'inverse, en fin d'excavation, le comportement de l'ouvrage est gouverné par la butée exercée dans la partie en fiche qui assure la stabilité de l'ouvrage. Les essais triaxiaux menés sur ces chemins font apparaître des modules élastiques plus élevés (75 à $100 \mathrm{MPa}$ selon l'état de contrainte initial). La prise en compte avec le modèle de Mohr-Coulomb de cette variabilité des modules permet une meilleure modélisation des différentes phases d'excavation (Fig. 10).

Ces résultats ne sont que quelques exemples des possibilités qu'offre l'utilisation conjointe de la modélisation physique et de la modélisation numérique. La complémentarité de ces deux méthodes doit être mise à profit de manière plus constante à l'avenir, à la fois pour fournir des données pour la validation des modèles numériques, mais aussi pour aider à la conception des modèles physiques.

\section{Conclusions}

Cet examen rapide des principaux programmes d'étude sur modèles réduits centrifugés conduits au LCPC durant ces quinze dernières années permet d'illustrer le spectre d'application de ces modèles et leur apport lors de la conception et du dimensionnement des ouvrages géotechniques, en particulier dans le domaine maritime. On assiste par ailleurs à une évolution des thèmes d'étude vers les problèmes de géotechnique de l'environnement, de transfert de polluants [30] et de simulation de séismes [31].

Les modèles réduits permettent de contribuer au dimensionnement des fondations, en particulier pour les ouvrages maritimes où les essais sur ouvrage réel sont plus difficiles, voire impossible à réaliser. Ils présentent l'avantage de pouvoir instrumenter aisément les fondations et le massif de sol, de conduire si besoin les essais jusqu'à la rupture et de mener des études paramétriques à faible coût.

En parallèle avec ces programmes d'études d'ouvrages géotechniques, une part importante de l'activité reste consacrée à l'analyse des conditions de similitude. Les objectifs sont une meilleure connaissance des limites de la méthode permettant dans certains cas d'étendre les possibilités et d'améliorer la pertinence des modèles.

Les modèles physiques, comme les modèles numériques, ne simuleront jamais les ouvrages réels sous tous les aspects de leur comportement, mais permettent d'étudier le comportement global au travers d'études paramétriques contrôlées. Ils doivent toujours être élaborés en fonction de questions et d'objectifs limités et bien définis.

\section{Références}

[1] J. Garnier, Modélisation physique en mécanique des sols : Application aux recherches sur les fondations et autres ouvrages géotechniques, Mémoire HDR, Université de Nantes, 2001a, 199 p.

[2] J. Garnier, Modèles Physiques en Géotechnique : État des connaissances et développements récents, $1^{\mathrm{e}}$ Conférence Coulomb, Paris CNIT La Défense, 2001b

[3] G. Pilot, Centrifugation de modèles réduits d'ouvrages en terre et de fondations, Rapport de recherche LCPC, 48, octobre 1975, $41 \mathrm{p}$. 
[4] J.-F Corté, J. Garnier, Une centrifugeuse pour la recherche en géotechnique, Bull. liaison laboratoires des Ponts et Chaussées 146 LCPC (1986) 5-28

[5] J. Garnier, F. Derkx, L.-M. Cottineau, G. Rault, Études géotechniques sur modèles centrifugés, Évolution des matériels et des techniques expérimentales, Bull. liaison des labo. Ponts et Chaussées 223 LCPC (1999) $27-50$

[6] J.-F. Corté (ed.). Int. Conf. Centrifuge 88, Paris, 2527 avril, Balkema, 1988, 610 p.

[7] H.Y Ko., F.G Lean (eds), Int. Conference Centrifuge 91, Boulder, Balkema, 1991, 633 p.

[8] C.F. Leung, F.H Lee, E.T.S Tan (Eds), International Conference Centrifuge 94, Singapore, 31 Aug.-2 Sep., ISBN 905410352 3, Balkema, 1994, 836 p.

[9] T. Kimura, O. Kusakabe, J. Takemura (Eds), International Conference Centrifuge 98, Tokyo, Balkema, ISBN 905410986 6, 1998, 919 p.

[10] R. Phillips, P.J. Guo, R. Popescu, Physical modelling in Geotechnics, ICPMG'02, ISBN 9058093891, Balkema, 2002, 1025 p.

[11] A. Bouafia, Modélisation des pieux chargés latéralement en centrifugeuse, thèse université de Nantes et ENSM, Nantes, 1990

[12] S. Mezazigh, Étude expérimentale de pieux chargés latéralement : proximité d'un talus et effet de groupe, Thèse de Doctorat, ECN, 28 Nov. 1995, 217 p. + Annexes $55 \mathrm{p}$.

[13] D. Remaud, Pieux sous charges latérales : étude expérimentale de l'effet de groupe, thèse de doctorat de l'Université de Nantes, 1999

[14] M. Hajialilue Bonab, J.-L. Chazelas, D. Levacher, Simulation du comportement d'un pieu isolé soumis à un choc latéral en centrifugeuse, Conférence Caquot, Paris, octobre 2001

[15] F. Rosquoët, Y. Canepa, J. Garnier, L. Thorel, Étude expérimentale sur modèles réduits centrifugés de l'effet des cycles sur l'interaction sol-pieu, $16^{\mathrm{e}}$ Congrès Français de Mécanique, Nice, 1-5 Sept. 2003, 8 p.

[16] R.D. Raines, J. Garnier, Physical modelling of suction piles in clay, OMAE Conference, Vancouver, 20-25 June, 2004, pp. 1-11

[17] J.D. Murff, The geotechnical centrifuge in offshore. Offshore Technology Conf., OTC 8265, 1996, 15 p.

[18] E.C. Cluckey, M. Morrison, A centrifuge and analytical study to evaluate suction caissons for TLP applications in the Gulf of Mexico, Performance of deep foundations, ASCE, Dallas, 1993, pp. 141-155

[19] E.C. Cluckey, M. Morrison, J. Garnier, J.-F.Corté J.F, The Response of Suction Caissons in Normally Consolidated Clays to Cyclic TLP Loading Conditions, 27th Annual OTC, Houston, Texas, USA, 1-4 May, 1995, pp. 909-918
[20] M. Morrison, E.C. Cluckey, J. Garnier, Behavior of suction caissons under static uplift loading, Conf. Int. Centrifuge 94, Singapour, Août/Sept., 1994, pp. 823-828

[21] J. Garnier, G. Rault, L.-M Cottineau, Applications de la modélisation physique au domaine de l'offshore, AUGC, Nantes, 1995, pp. 129-136

[22] A. Puech, J.-P. Iorio, J. Garnier, P. Foray, Experimental study of suction effects under mudmat type foundations, $4^{\mathrm{e}}$ Conf. Canadienne Génie Géotechnique Marin, St-John's, Newfoundland, Vol. 3, 1993, pp. 1062-1080

[23] J. Garnier, A. Pecker, Use of centrifuge tests for the validation of innovative concepts in foundation engineering, 2nd Int. Conf. Earthquake geotech. Engng., Lisbonne, 1999, 7 p.

[24] A. Pecker, Pont de Rion-Antirion : fiabilité et conception parasismique des fondations, la sécurité des grands ouvrages, Presses ENPC, 2000, pp. 21-37

[25] J.-P. Teyssandier, J. Combault, A. Pecker, Rion-Antirion, le pont qui défie les séismes, La recherche, 334 (2000) $42-46$

[26] C. Gaudin, Modélisation physique et numérique d'un écran de soutènement autostable. Application à l'étude de l'interaction écran-fondation, thèse Université de Nantes, ED MTGC, 2002, 410 p.

[27] C. Gaudin, J. Garnier, G. Rault, P. Gaudicheau, Use of a robot for in-flight excavation in front of an embedded wall. Int. Conf. Physical Modelling Geotechnics. ICPMG'02 July 10-12 St. John's, NF, Canada, 2002a, pp. $77-82$

[28] C. Gaudin, Y. Riou, H. Popa, J. Garnier, Numerical modelling of centrifuge test on embedded wall, NUMGE 02, Mestat (Ed.), Presses ENPC/ LCPC, ISBN 2720860042, Paris 4-6 Sept. 2002c, pp. 689-696

[29] C. Gaudin, J.-J. Serratrice, L. Thorel, J. Garnier, Caractérisation du comportement d'un sol par essais triaxiaux pour la modélisation numérique d'un écran de soutènement, Symp. Int. PARAM 02, Magnan (ed.), Paris, 2-3 Sept., ISBN 2-7208-6003-4, 2002b, pp. 385-392

[30] J. Garnier, L. Thorel, E. Haza, (Eds), Physical modelling and testing in environmental geotechnics, Int. Symposium, La Baule, May 15-17, ISBN 2-7208-6050-6, 2000, $392 \mathrm{p}$.

[31] J.-L. Chazelas, Quinze ans de simulation sismique en centrifugeuse dans le monde, $5^{\mathrm{e}}$ colloque national AFPS'99, ENS Cachan, 19-21/09/1999, pp. 163-170

[32] L. Thorel, J. Garnier, G. Rault, A. Bisson, Étude de la résistance à l'arrachement sous charge verticale des ancres à effet de succion, J. Nat. de Géotechnique et de Géologie de l'Ingénieur, 8-9 Oct. 02, Nancy, 2002, 12 p. CDROM

[33] A. Pecker, Apport des essais en centrifugeuse au dimensionnement d'une fondation de pont. Coll. Mécanique et Géotechnique, Jubilé Scient. P. Habib, 19 mai 1998, LMSÉcole Polytechnique, 1998, 11 p. 